\title{
Comparative genomic analysis of the compound Brassica napus Rf locus
}

\author{
Lydiane Gaborieau and Gregory G. Brown ${ }^{*}$
}

\begin{abstract}
Background: The plant trait of cytoplasmically-inherited male sterility (CMS) and its suppression by nuclear restorerof-fertility $(R f)$ genes can be viewed as a genetic arms race between the mitochondrial and nuclear genomes. Most nuclear Rf genes have been shown to encode P-type pentatricopeptide repeat proteins (PPRs). Phylogenetic analysis of P-class PPRs from sequenced plants genomes has shown that Rf-proteins cluster in a distinct clade of P-class PPRs, RFLPPRs, that display hallmarks of positive evolutionary selection. Genes encoding RFL-PPRs (RFLs) within a given plant genome tend to be closely related both in sequence and position, but a detailed understanding of how such speciesspecific expansion occurs is lacking. In the canola, (oilseed rape) species Brassica napus, previous work has indicated the nuclear restorer genes for the two native forms of CMS, Rfn (for nap CMS) and Rfp (pol CMS), represent alternate haplotypes, or alleles, of a single nuclear locus.

Results: Fine genetic mapping indicates that Rfn does indeed localize to the same genomic region as Rfp. We find this region is enriched in RFL genes, three of which, based on their position and expression, represent potential candidates for Rfn; one of these genes, designated PPR4, is a preferred candidate in that it is not expressed in the nap CMS line. Comparison of the corresponding regions of the genomes of B. rapa, B. oleracea, Arabidopsis thaliana and A. lyrata provides insight into the expansion of this group of RFL genes in different lines of evolutionary descent.

Conclusions: Unlike other nuclear restorer loci containing multiple RFL genes, the RFL genes in the Rf region of $B$. napus are not present in tandem arrays but rather are dispersed in genomic location. The genes do not share similar flanking non-coding regions and do not contain introns, indicating that they have duplicated primarily through a retrotransposition-mediated process. In contrast, segmental duplication has been responsible for the distribution of the 10 sequences we annotated as RFL genes in the corresponding region of the A. lyrata genome. Our observations define the Brassica Rf locus and indicate that different mechanisms may be responsible for the proliferation of RFL genes even among closely related genomes.
\end{abstract}

Keywords: Cytoplasmic male sterility, Nuclear fertility restoration, Pentatricopeptide repeat, RFL gene, Retrotransposition, Nomadic gene

\section{Background}

Cytoplasmic male sterility (CMS) is a widespread, maternally inherited trait of flowering plants that results from the expression of novel genes in the mitochondrial genome. The novel genes are unique for each type of CMS and often are chimeric in structure, consisting of segments of standard mitochondrial genes fused, in frame, to other open reading frames that bear no resemblance to known functional genes [1]. The novel gene products associated with CMS are inner membrane proteins

* Correspondence: greg.brown@mcgill.ca

Department of Biology, McGill University, 1205 Doctor Penfield Ave., Montreal, QC H3A 1B1, Canada whose presence is thought to compromise mitochondrial function, although the specific mechanisms whereby this leads to an abrogation of functional pollen formation, are, with the exception of a few examples (e.g. [2-4]), not clear.

The CMS phenotype is often masked by the presence of nuclear restorer genes. These genes are specific to each form of CMS and in general act to down-regulate, at the post-transcriptional level, the expression of the cognate novel CMS-causing mitochondrial genes. Consequently CMS can often only be revealed through wide intraspecific or interspecific crosses. CMS is also revealed in nature via gynodioecy, populations consisting 
of a mixture of female (i.e. male sterile) and hermaphroditic plants. Theoretical studies have indicated that maternally inherited male sterility can, under certain circumstances, spread in populations; if the frequency of male sterile individuals then becomes sufficiently high, pollen becomes scarce, a condition favoring the counterselection of nuclear restorers that suppress the malesterility [5-7]. This type of interaction can be viewed in the context of genomic conflict: the selective interests of the uniparentally inherited mitochondrial genome oppose those of the biparentally-inherited nuclear genome [6]. Such genomic conflicts are often characterized as a genetic arms race: the appearance of a new male-sterility mitochondrial gene will drive the appearance of a corresponding restorer gene, analogous to the "gene-for-gene" selection of new host resistance genes in response to new pathogen races $[8,9]$.

A number of nuclear restorers have been isolated in recent years [10-18]. Most of these have been shown to encode proteins composed of tandem repeats of a degenerate 35 amino acid sequence, the pentatricopeptide repeat (PPR) [19]. Most eukaryotic genomes harbor only a few PPR-encoding genes, but in plants this gene family has greatly expanded, and contains plant specific forms with repeats that are longer and shorter than the canonical 35 amino acid motif [20]. Most plant PPR proteins are targeted to the mitochondria and chloroplasts, and bind to specific RNA substrates, mediating numerous aspects of post-translational gene expression including splicing, nuclease processing, editing and translation [21]. Restorer PPR proteins are largely composed exclusively of core repeat motifs as defined by the P-type PPR subfamily, and comprise a distinct phylogenetic clade of such proteins, Rf-like PPRs, whose corresponding coding sequences are designated as RFL genes [22]. Comparison of the RFL genes within a given species reveals evidence of positive evolutionary selection, consistent with the premise of genomic conflict [22-24]. Moreover, the position of certain $R f$-like PPR genes are not conserved between otherwise closely-related, syntenic chromosomal regions [23] giving these genes a "nomadic"-like character.

Two native CMS systems, nap and pol, are known to occur in the oilseed rape (canola) species Brassica napus. The causative factor for the "Polima" or pol CMS, orf224, is a novel CMS-associated mitochondrial gene in which the promoter and first 58 codons of the atp 8 gene are fused to a unique sequence bearing little similarity to other known sequences [25]. nap CMS is specified by orf 222 , a chimeric gene that is similar but not identical over its entire length to orf224 [26]. The two genes are located in different positions on the mitochondrial genome: orf 224 is situated upstream of the atp 6 gene, while orf 222 is located upstream of $\operatorname{nad} 5$ exon c. The mitochondrial genome of the male fertile B.napus cytoplasm, cam, is identical to that found in one of the progenitor species, $B$. rapa (formerly campestris, see below) and lacks both orf 222 and orf224. The restorers for the pol and nap systems, $R f p$ and $R f n$, respectively, each down-regulate the expression of their cognate CMSassociated mitochondrial genes by mediating RNA cleavage events within unique regions of the corresponding transcripts [25, 26]. The $R f n$ allele is also associated with additional RNA cleavage events in the coding regions of nad4 and $c c m F_{N 2}$ (formerly ccl1-l $[27,28]$ ) which are not observed in plants homozygous for the $R f p$ allele or for the non restoring, or universal maintainer genotype $r f$ [29]. Indeed, $R f n$ and $R f p$ represent distinct alleles or haplotypes of a single nuclear locus, and it has not been possible to separate these genes or their associated mtRNA cleavage properties via genetic crosses involving the three nuclear and cytoplasmic genotypes [8]. Together, these characteristics indicate that two CMSrestorer systems have evolved in the relatively recent evolutionary past, and that $B$. napus CMS systems therefore offer an attractive model for understanding the molecular events underlying the evolution of CMS-restorer gene systems [29].

Further progress towards this goal requires the identification and characterization of $R f p$ and $R f n$ genes and an understanding of the evolution of the genomic regions surrounding them. The complexity of the amphidiploid $B$. napus genome has posed a key challenge in the accomplishment of this goal. Following the evolutionary divergence of the Brassica and Arabidopsis lineages about 35 Mya [30, 31] polyploidization events gave rise to an ancestral Brassica genome in which most Arabidopsis thaliana regions were present as 3 co-linear copies [32]. Modern diploid Brassica genomes reflect further genomic rearrangement, gene loss and gene duplication and show fragmented co-linearity with Arabidopsis, with each co-linear region being represented, on average, in three copies. B. napus is the product of a relatively recent interspecific hybridization between two such Brassica species, B. rapa (source of the A genome) and B. oleracea (source of the $C$ genome), which diverged from one another between 2.6 and 4.2 Mya [33].

We have previously introgressed the $R f p$ gene into the $B$. rapa genome and have fine-mapped the gene to a segment of the B. napus genome co-linear with A. thaliana chromosome 1 coordinates 4.28-4.40 [34, 35]. We report here our genetic localization of the $R f n$ gene and the molecular characterization of the genome regions surrounding it. We identify a preferred candidate for $R f n$. In addition, by analyzing the positions and sequence relationships between the RFL genes in related regions of Arabidopsis and Brassica genomes, we are able to 
draw inferences regarding the molecular events through which this gene family has expanded and the $R f$ locus evolved.

\section{Results}

Identification of a BAC clone corresponding to the $R f p / R f n$ region

A $B$. rapa cosmid clone containing a polymorphism tightly linked to $R f p$, a SNP in the gene corresponding to A. thaliana At1g12910 at chromosome 1 coordinate 4.395 [35], was recovered, sequenced and used as a source of probes to screen a B. napus bacterial artificial chromosome (BAC) library derived from a line with the $R f n$ genotype (see Methods) at the restorer locus. A single BAC, of approximately $180 \mathrm{~kb}$, designated NO202E11, containing a sequence identical to the corresponding region of the cosmid probe was selected and sequenced. Sequence analysis showed that the BAC contained a sequence highly similar to the At1g12910 gene with the $R f n$-linked allele of the Atg12910-orthologous SNP. Since our previous studies indicated $R f p$ and $R f n$ are different haplotypes of the same genomic region, we considered this BAC to likely be anchored in the $R f n$ region. The BAC sequence was found to be collinear with the region of the $A$. thaliana genome extending from chromosome 1 coordinates 4.27-4.47 and with the region of the B. rapa genome extending from chromosome A09 coordinates $42.18 \mathrm{Mb}$ to $41.79 \mathrm{Mb}$. Dot matrix visualization of the synteny between the sequenced BAC and the B. rapa and A. thaliana genomic regions is shown in Figs. 1a and 1b, respectively.

\section{High resolution mapping of the $R f n$ gene}

The premise that $R f p$ and $R f n$ are alleles or closely linked alternative haplotypes of the same genetic locus is based on relatively rough genetic mapping and genetic crosses in which the transcript modification activities associated with the two genes have been found to be mutually exclusive [8]. We would therefore expect that higher resolution genetic mapping studies should confirm that $R f n$ does, indeed, co-localize with the region in the selected BAC. We constructed a $\mathrm{BC} 1$ mapping population, genotyped individuals with SNPs from the region of $B$. napus chromosome A09 corresponding to $B$. rapa coordinates $39.75 \mathrm{Mb}$ to 43 . $27 \mathrm{Mb}$. One of these SNPs, localized at coordinate $42.13 \mathrm{Mb}$, fell within the BAC sequence. Specific information on the SNPs that were polymorphic between the mapping parents of the cross is provided in Additional file 1: Table S1.

The nap CMS phenotype is leaky in a temperaturedependent, cultivar-specific manner [36, 37], a challenge for the precise mapping of $R f n$. The choice of the $R f n$ parent for the mapping cross is therefore critical. Based on our previous work, we knew that utilizing the cultivar "Karat" could provide a BC1 mapping population that would allow us to satisfactorily distinguish between CMS and fertility restored progeny [8]. To derive our $\mathrm{BC} 1$ population, two $\mathrm{F} 1$ individuals generated by pollinating CMS plants $(r f / r f[n a p])$ with Karat $(R f n / R f n[n a p])$ were crossed back as females to the Karat parent. Of 293 individual BC1 plants, 146 were unambiguously scored as fertile (Rfn/rf [nap]) and 147 as male sterile $(r f / r f[n a p])$. The floral phenotypes of the parental and
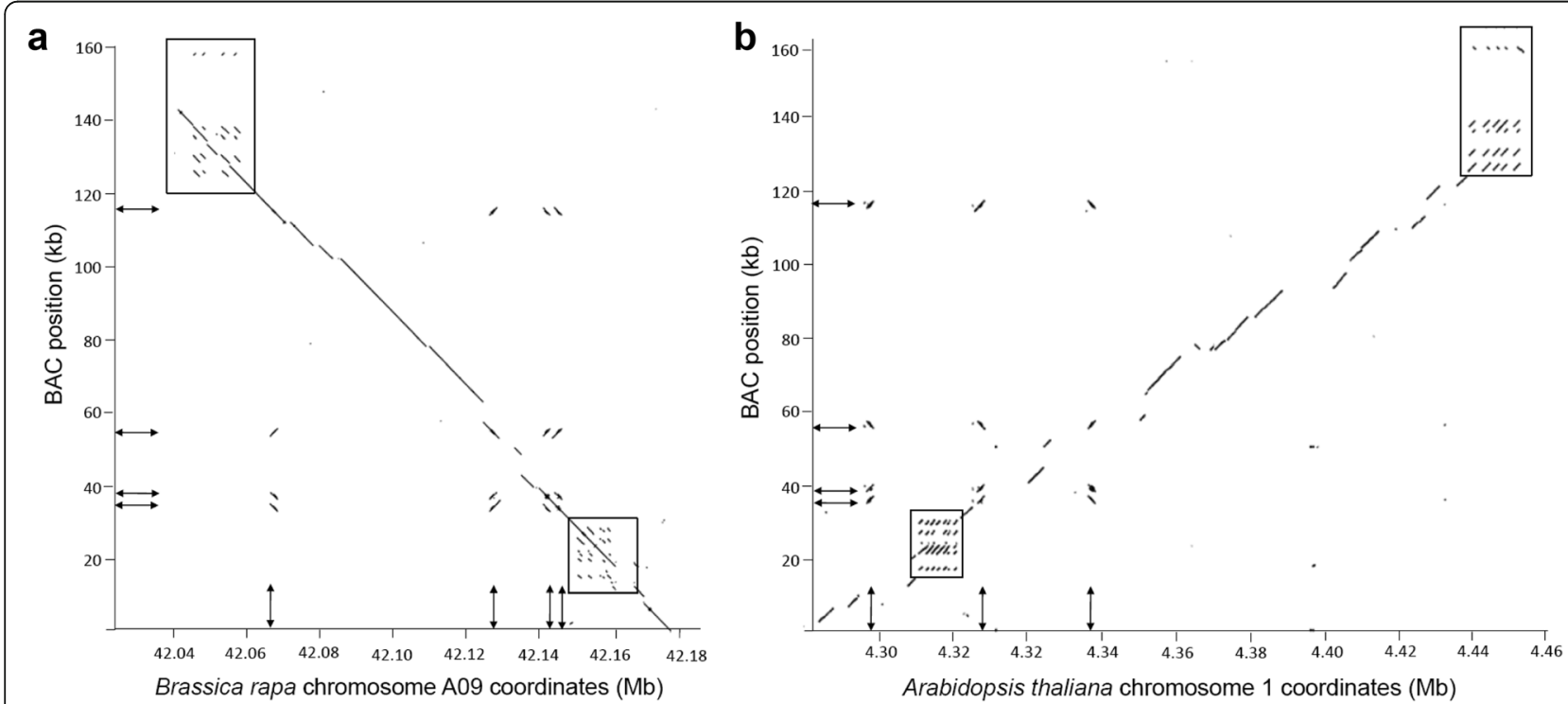

Fig. 1 Dot matrix alignments of the sequence of a bacterial artificial chromosome (BAC) anchored in the Rfp region with the syntenic regions of B. rapa chromosome A09 (a) and A. thaliana chromosome 1 (b). Boxed regions indicate regions encoding short repeated gene/pseudogene sequences. The arrows indicate repeated regions showing similarity to four and three distinct sites in B. rapa and A. thaliana respectively 
fertile and sterile progeny are illustrated in Fig. 2a. Genotyping of the population, as illustrated in Fig. 2b, allowed us to map the $R f n$ gene to the region of $B$. napus chromosome BnA09 containing the selected BAC, confirming that $R f p$ and $R f n$ were closely linked alleles. The single marker positioned within the BAC was perfectly linked to $R f n$. Because both $R f p$ and $R f n$ are located within this chromosomal region we henceforth refer to it as the B. napus $R f$ locus.

To more precisely localize $R f n$ we identified additional polymorphic molecular markers mapping within the region delimited by the SNPs. Primers designed to amplify genomic regions extending across introns in genes located between the SNPs most proximal to $R f n$ identified two intron length polymorphisms (ILPs, [38]), amplification products that differed in size between the two parents of the mapping population. When no noticeable amplicon length difference was evident, the products were further subjected to restriction cleavage to reveal cleaved amplified polymorphisms (CAPS, [39]). This strategy allowed four additional polymorphic markers anchored in the targeted region to be identified. These markers were then used to genotype individuals in which recombination had occurred between the closest flanking SNPs. This strategy allowed us to delimit the $R f n$ containing region to the segment of corresponding $B$. rapa chromosome A09 coordinates $41.68-42.58 \mathrm{Mb}$.

\section{Characteristics of the $B$. napus $R f$ locus}

Several interesting features were revealed through dot matrix visualization of the synteny between the sequenced BAC and the B. rapa and A. thaliana genomic regions (Figs. 1a and 1b, respectively). Regions at each end of the BAC, roughly located at positions 10-30 and 120-140 kb, showed similarity to sets of short repeated sequences, shown as boxed regions on the figures. Detailed comparative annotation of the genes in the BAC with the corresponding portions of the $B$. rapa and $A$ thaliana (Additional file 2: Table S2) indicated that the repeated sequences corresponded to sets of directly repeated genes and/or pseudogenes encoding thionin (PR-13) proteins (positions 10-13 kb) in one case and Cytochrome $\mathrm{P}_{450}$ Cyp2 proteins (positions 120-140 kb) in the other. Comparative annotation with the more recently released $B$. napus cv. "Darfur" chromosome BnA09 [40] indicated that a sequence inversion has taken place in $B$. napus by which the sequence extending from $B$. rapa chromosome A09 coordinates 41.8042.04 $\mathrm{Mb}$ is inverted (Additional file 3: Table S3). The inverted region is flanked by sequence spans encoding Cytochrome $\mathrm{P}_{450}$ Cyp2 and F-box domain encoding genes. This rearrangement appears as a gap in synteny from BAC coordinates $120-140 \mathrm{~kb}$ in both the $B$. rapa and $A$, thaliana plots. At around coordinate $160 \mathrm{~kb}$, near the very end of the BAC, sequence similarity is

a

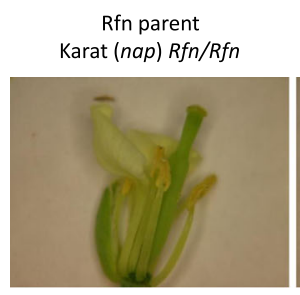

CMS parent 70/7406 (nap) rf/rf

BC1 fertile

(nap) $R f n / r f$

BC1 male-sterile

(nap) $r f / r f$
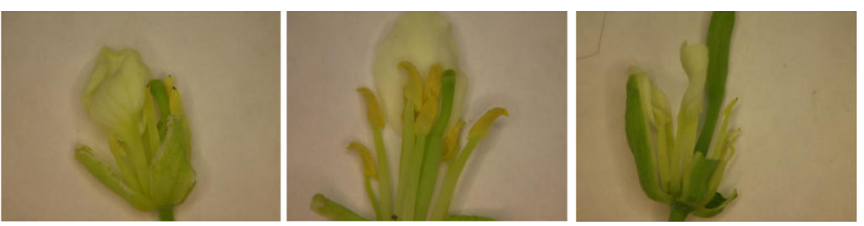

b

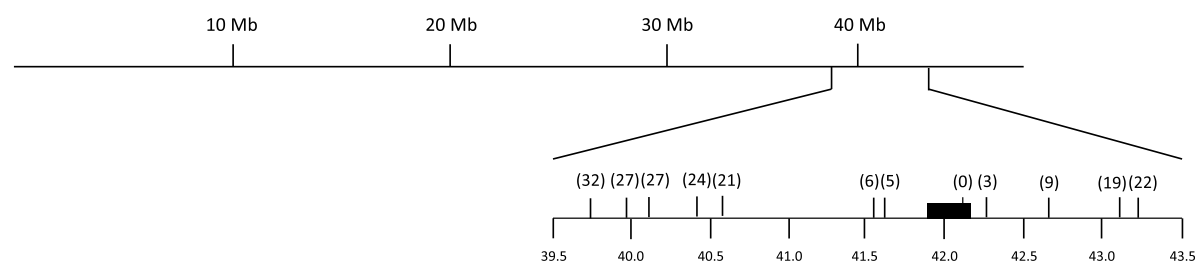

Fig. 2 Mapping of the Rfn gene on chromosome A09. a Flowers from the parents and BC1 progeny of the mapping cross. Petals have been removed to allow display of anther morphology. Cytoplasm is designated in parentheses. The male parent of the cross and fertile progeny heterozygous for Rfn (Rfn/rf) have anthers with normal morphology and shed abundant pollen. The male sterile (CMS) parent and BC1 progeny homozygous for the recessive maintainer allele (rf/rf) have stamens with short filaments and underdeveloped anthers that shed little or no pollen. b Location of Rfn on chromosome A09. The region of the B. rapa chromosome chosen as range for targeted mapping extending from coordinates 39.5 to $43.5 \mathrm{Mb}$ is expanded to illustrate the mapping results. Numbers in parentheses indicate the number observed recombination events between a marker at that location and the Rfn gene. The filled rectangle indicates position of the BAC spanning the region delimited in Rfp mapping experiments. No recombination was observed between the single marker located within the BAC and the Rfn gene 
observed between the $\mathrm{BAC}$ and the Cytochrome $\mathrm{P}_{450}$ Cyp2 genes, but in reverse orientation, as expected in the case of an inversion. Key features of the differences between the BAC, B. rapa and B. napus cv. "Darfur" genomes are illustrated in Fig. 3.

\section{Rf-like PPR genes in the B. napus Rf locus}

Another striking feature of the dot matrix comparisons was the presence of sequences located near BAC coordinates 34, 38, 52 and $118 \mathrm{~kb}$ (illustrated by double headed arrows in Figs. 1a and 1b) that mapped to four corresponding sites in B. rapa chromosome A09. In the Arabidopsis genome, only three of the four sites were located at corresponding positions; no Brassica sequence was found to correspond to the site located near the 4.295 Mb coordinate on Arabidopsis chromosome 1. Inspection of the Brassica repeat sequences indicated that they all corresponded to regions encoding highly similar RFL PPR genes predicted to be targeted to the mitochondria, which we designated as PPR1-4.

Because the genetically defined limits of the $R f n$ region extended beyond the boundaries of the BAC, we searched the corresponding region of the $B$ rapa chromosome 9 for additional RFL PPRs that could serve as candidates for $R f n$. We were able to identify two more such genes, one B.rara.I05036, located between coordinates 41.777 and $41.776 \mathrm{Mb}$, and the other, B.rara.I05115, between coordinates 42.211 and $42.213 \mathrm{Mb}$. Orthologous PPR genes at corresponding locations were found to be present on B. napus "Darfur" chromosome BnA09 and are designated Bn036 (coordinates 31.334-31.341 Mb) and Bn115 (31.797-31.806 Mb). Both of these genes encode products predicted to be targeted to the mitochondrion.
Notably, Bn036 is located roughly $100 \mathrm{~kb}$ from the flanking marker 4.4BB but $300 \mathrm{~kb}$ from the RFL genes in the BAC. No genes encoding PPR proteins other than RFL genes were detected in the region. A preliminary phylogenetic analysis (Additional file 4: Figure S1) indicated that five of the six genes clustered with three A. thaliana $R F L$ genes located on the long arm of chromosome 1, AtRFL2 (At1g12300), AtRFL3 (At1g12620) and At1g12775 (reannotated At1g12770 or AtRFL25). The sixth gene PPR2, clustered within a neighboring branch with its ortholog AtRFL4 (At1g12700). PPR3 and AtRFL25, like PPR2 and AtRFL4, are located at matching positions in their corresponding chromosomes.

A comparison of the relative positions of the genes in the A. thaliana and B. rapa/B. napus A genomes is presented in Fig. 4. The phylogenetic analysis indicated that all of the Brassica genes represented Rflike PPRs, as did three of the four Arabidopsis genes; a non-RFL A. thaliana PPR gene, At1g13030, is found at a site close to but not precisely matching the Brassica RFL PPR4 gene at coordinate $118 \mathrm{~kb}$ in the BAC. One of the genes, At1g12700 (AtRFL4, [41]) located at a matching position in both genomes, is known to encode a mtRNA processing factor, RPF1, which confers nuclease cleavage events on nad4 transcripts, which are also a target of the Brassica $R f n$ gene. These observations revealed six RFL B. napus PPR genes that could serve as candidates for $R f n$. One of the genes, PPR4, has been previously proposed as a candidate for $R f p$ on the basis of fine mapping data [42]. None of the Brassica PPR genes were predicted to contain an intron, an observation confirmed by RT-PCR analysis of floral transcripts (see below).

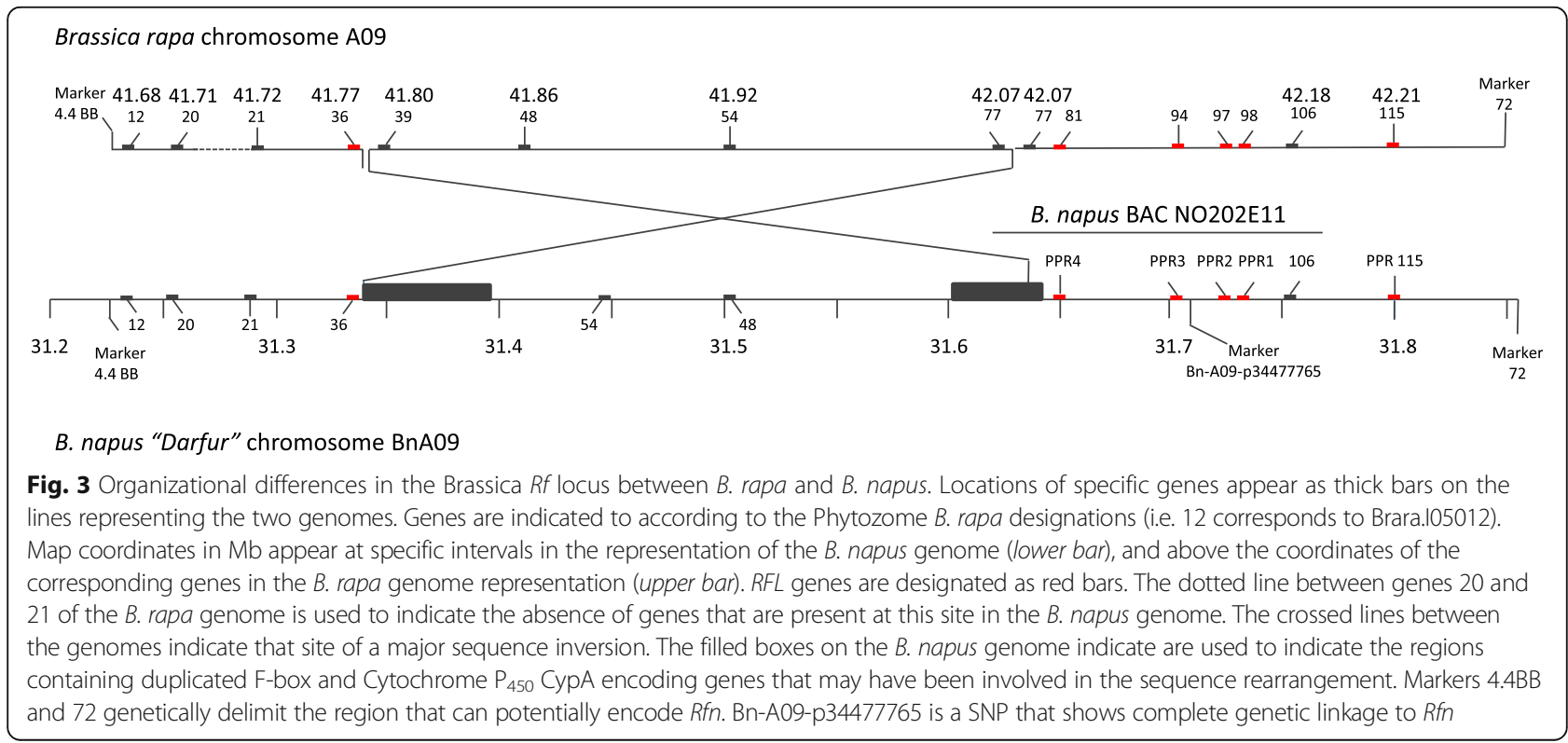




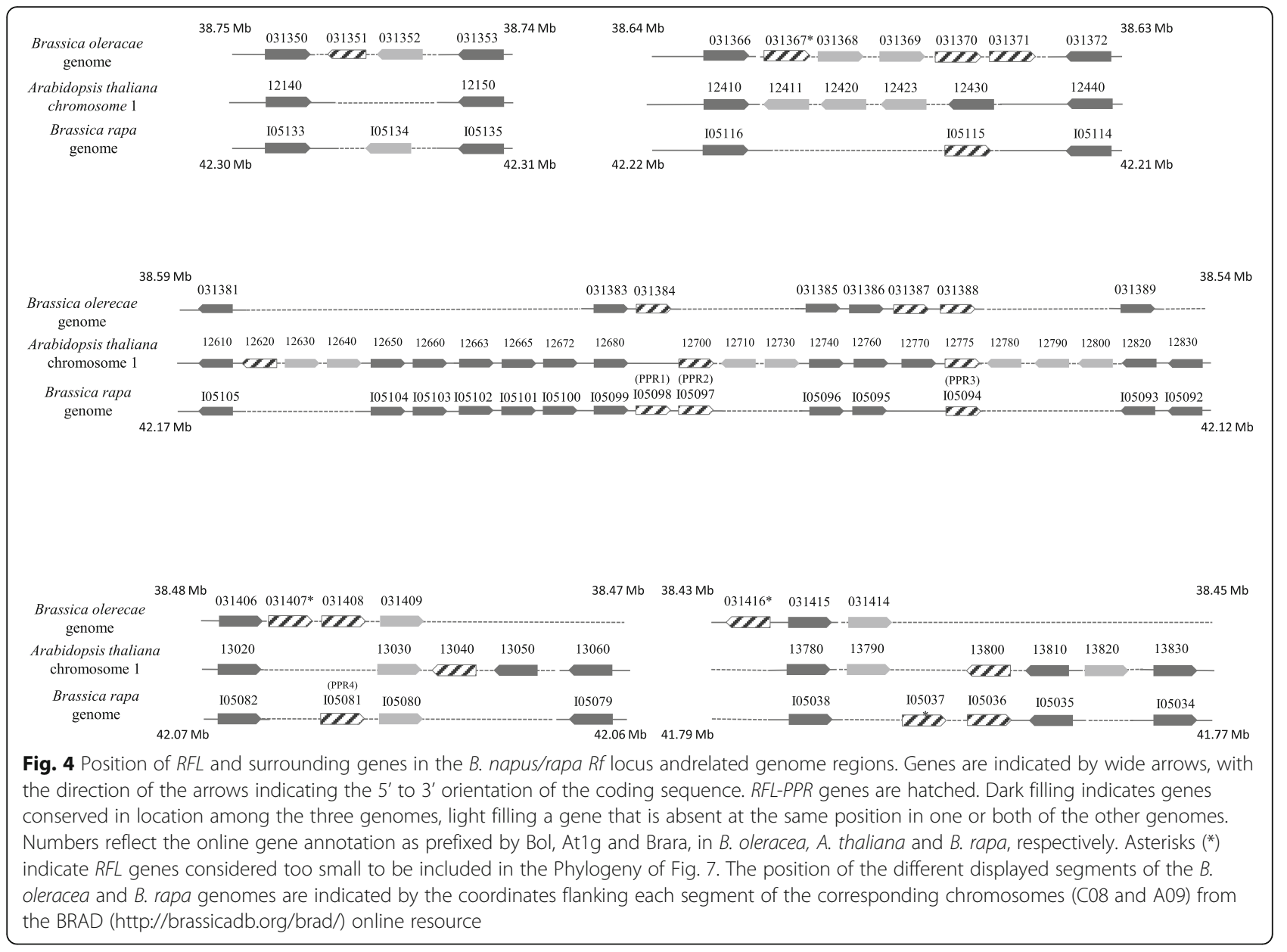

\section{Expression of the Rf-region RFL genes in nap CMS and fertility restored plants}

The observation that $r f$-PPR592, the non-restoring allele of the petunia restorer, Rf-PPR592, is not expressed but is otherwise similar to the restorer suggested that expression differences among different candidate PPR genes could be used as a tool to prioritize candidates for further analysis of restoration function. We used RTPCR to examine the expression of the six candidate RFL genes located within the Brassica $R f$-locus. As shown in Fig. 5, we did not detect expression of Bn115 in floral buds of either CMS or nuclear restored plants. Of the remaining 5 B. napus Rf-region RFL genes, expression of one, PPR4, was detected in the buds of nuclear fertility restored but not CMS plants; PPR4, is thus seen a strong candidate for Rfn. PPR1 and PPR3 both also show higher levels of expression in restored than in CMS flowers.

The sequences of the RT-PCR products, as shown in Additional file 5: Figure S2, provided further information relevant to the structures of the genes and their possible roles in nuclear fertility restoration. The sequences of the transcripts were co-linear with the corresponding genomic DNA sequence, indicating that, as expected, these genes lacked introns. Interestingly, a termination codon was found at nucleotide position 1119 in the coding sequence of the RT-PCR products of PPR2 from both the CMS and nuclear restored lines. This termination codon was not detected in genomic sequences of either B. rapa or the BAC NO202E11, and resulted from a one nucleotide insertion in the CMS and restorer sequences prior to the termination codon, followed by a two nucleotide insertion in the same sequences.

\section{Expansion of a family of Rf-like PPR genes within Brassica genomes}

The B. napus genome is derived from a recent interspecific hybridization event between the $C$ genome species $B$. oleracea and the A genome of $B$. rapa, two species which diverged in descent approximately 3.7 Mya. Because RFL PPR genes are known to be variable in chromosomal position between closely related genomes $[22,23]$, it was of interest to determine the position of the $R f n$ candidates and close paralogs in the B. oleracea $\mathrm{C}$ genome. To accomplish this we first identified close 


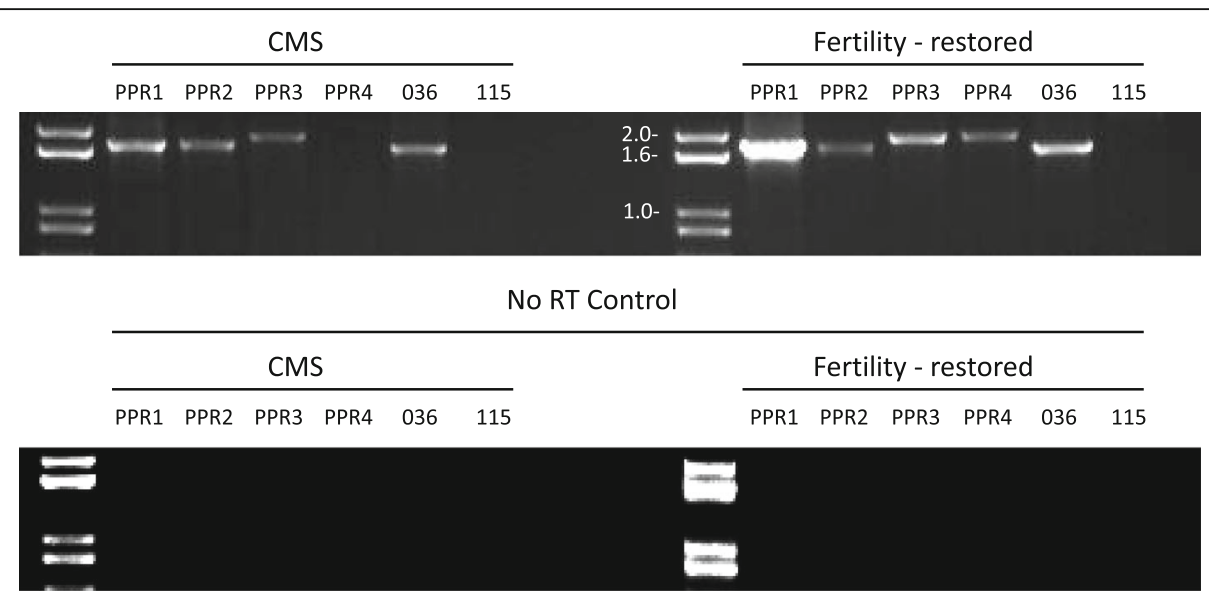

Fig. 5 Expression of $R f$ locus B. napus PPR genes. Total RNA was extracted from the CMS and restorer parents of the mapping cross and analyzed by RT-PCR. The lower panel shows the results of the experimental control, performed at the same time on the same group of samples, in which reverse transcriptase was omitted prior to PCR amplification of cDNA products

homologs of the different $B$. napus Rf region PPR genes on the $B$. oleracea genome using the blastn resource of the Brassica database (BRAD, [43]). We found a cluster of highly similar sequences between chromosome 8 coordinates 38.54 and $38.75 \mathrm{Mb}$, a region over which synteny was maintained with the corresponding regions of $A$. thaliana and B. rapa. The annotation of the region indicated that the homologous sequences corresponded to 10 highly related RFL-PPR genes. Eight of these genes lacked predicted introns, whereas two, Bol31351 and Bol31388, were each predicted to contain a single intron.

The relative position of PPR genes in the A. thaliana, $B$. rapa/napus (A) and $B$. oleracea (C) genomes in the region over which synteny is conserved among the three genomes is illustrated in Fig. 4. Seven of the $10 \mathrm{C}$ genome B. oleracea PPR genes are located a position corresponding their location in the A genome. In three cases, involving Bol31370/Bol31371, Bol31387/Bol31388 and Bol313407/Bol313408, a tandem pair of B. oleracea PPR genes is found at sites occupied by only a single PPR gene in the A genome. In one case a single B. oleracea gene, Bol31384, was found at a site containing two adjacent PPRs in the A genome. These observations indicated that the relative locations of the majority of $\mathrm{Rf}$ region PPR genes have not changed since the A/C genome divergence.

\section{Positional variation of RFL genes in the Rf-orthologous regions of two Arabidopsis genomes}

Because we observed some conservation of location between some $A$. thaliana RFL genes and Brassica $R F L$ genes in the Rf-region, it was of interest to determine to what extent this positional conservation could also be observed between the A.thaliana and A. lyrata genomes, which diverged from each other between 4 and 5 Mya. Fujii et al. [22] observed the RFL genes in the A. thaliana region orthologous to the Brassica Rf-region (Fig. 1a) fell into RFL subgroup 1, most of which are located between the 4.18 and $4.33 \mathrm{Mb}$ coordinates of chromosome 1. A. lyrata subgroup 1 genes similarly cluster within a $221 \mathrm{~kb}$ segment of the scaffold 1 genome assembly unit.

Dot matrix visualization of the similarities between these two Arabidopsis genomic regions revealed several interesting features of the positional relationships among these genes (Fig. 6) At the position corresponding to AtRFL2 (At1g12300), two highly similar genes (indicated by the double-headed arrows in Fig. 6a), designated in Fig. $6 \mathrm{~b}$ as AlyRFL1 and AlyRFL2 ${ }^{1}$, are found at the corresponding site in the A. lyrata genome. The 5' and 3' non-coding regions surrounding these genes are similar to one another, indicating that the two lyrata genes arose from a tandem duplication of a region encoding an AtRFL2-like gene. At the position corresponding to AtRFL3 (At1g12620), a tandem triplication of a similar gene was found at two different positions in the A. lyrata genome. This arrangement arose from the duplication of an approximately $14 \mathrm{~kb}$ region spanning the triplication and extending in the direction matching the centromere proximal side of $A$. thaliana chromosome 1 (Fig. 6a). Thus six RFL genes, which we designate as AlyRFL3-9, are found within a duplication spanning the region around AtRFL3. Another tandem duplication with sequence similarity to the AlyRFL1/ AlyRFL2 pair is found at a site corresponding to AtRFL4 (At1g12700). These observations suggest segmental duplication is the primary mechanism behind the proliferation of this family of $R F L$ genes in this region the $A$. lyrata genome. 


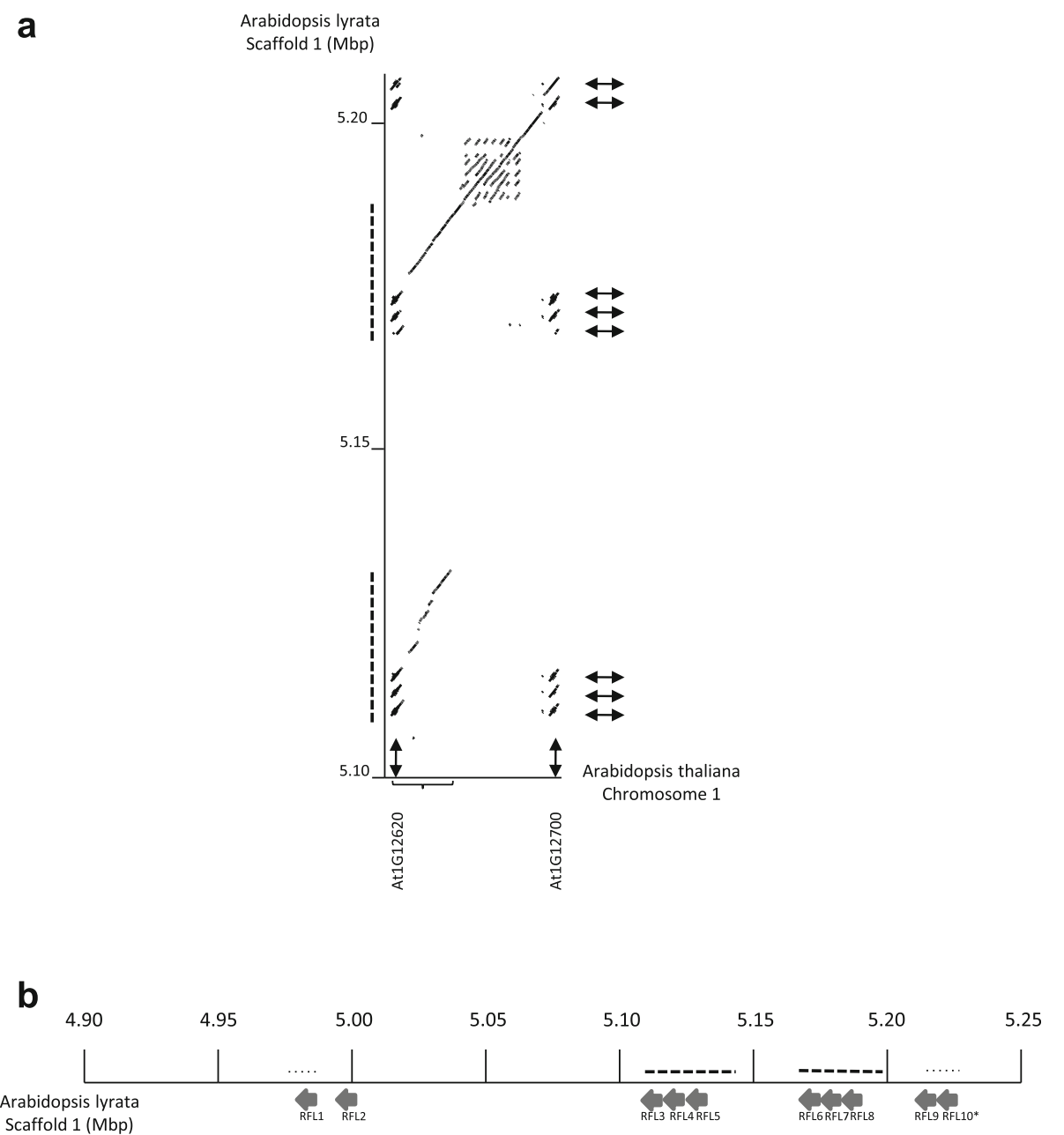

Fig. 6 RFL genes in a region of the A. lyrata genome orthologous to the region of the A. thaliana genome depicted in Figs. 1 and 3. a A dot matrix representation illustrating the duplication of a region containing 3 RFL genes at positions indicated by the double headed in the $A$. lyrata genome; all three genes and the surrounding sequences correspond to the AtRFL3 (At1g12620) region of $A$. thaliana. The bracket indicates the region of the Arabidopsis genome that is duplicated in A. lyrata (dotted lines along the $y$ axis). A second duplication occurs at the position of AtRFL4 (At1g12700); in this case the duplication involves the AtRFL2 gene (At1g12300). b Depiction of the analyzed segment of the A. lyrata genome. Arrows indicate the direction and orientation of the RFL genes. The dotted lines indicate duplicated segments: one containing three genes at the location corresponding to AtRFL3 and the other containing two genes apparently derived from AtRFL2, with one copy at the location of AtRFL2 and the other at the location of AtRFL4

\section{Phylogenetic relationships among Brassica and Arabidopsis RFL proteins}

We constructed a maximum-likelihood phylogeny to examine how the positional relationships among the various RFL genes reflected the sequence relatedness of the various encoded proteins. As shown in Fig. 7, all of the RFL proteins encoded in the Rf-syntenic regions of the different genomes formed a single monophyletic cluster encompassing the Arabidopsis subgroup 1 RFL proteins [22], and excluding radish Rfo/PPRA, their closest Arabidopsis homolog, AtRFL18, as well as the petunia Rf-PPR592 restorer protein and its non-restoring homolog (bootstrap support values of 0.90 and 1.00, respectively). Most of the Brassica proteins fell into a distinct cluster most closely related to the Arabidopsis branch containing AtRFL1-3. The exception were those proteins encoded by genes located at the same position as AtRFL4 (At1g12700), PPR2 and Brara.I05097; these formed a distinct phylogenetic cluster (bootstrap support 1.00), suggesting that these Arabidopsis and Brassica proteins have descended from a common ancestor located at the same position in the ancestral genome.

Within the major Brassica clade, proteins in a common genomic location generally clustered together in the tree. The major exception concerned the B. oleracea genes Bol03187 and Bol03188. These proteins formed a cluster distinct from that of their positional counterparts, the B. napus PPR3 proteins, which clustered with 


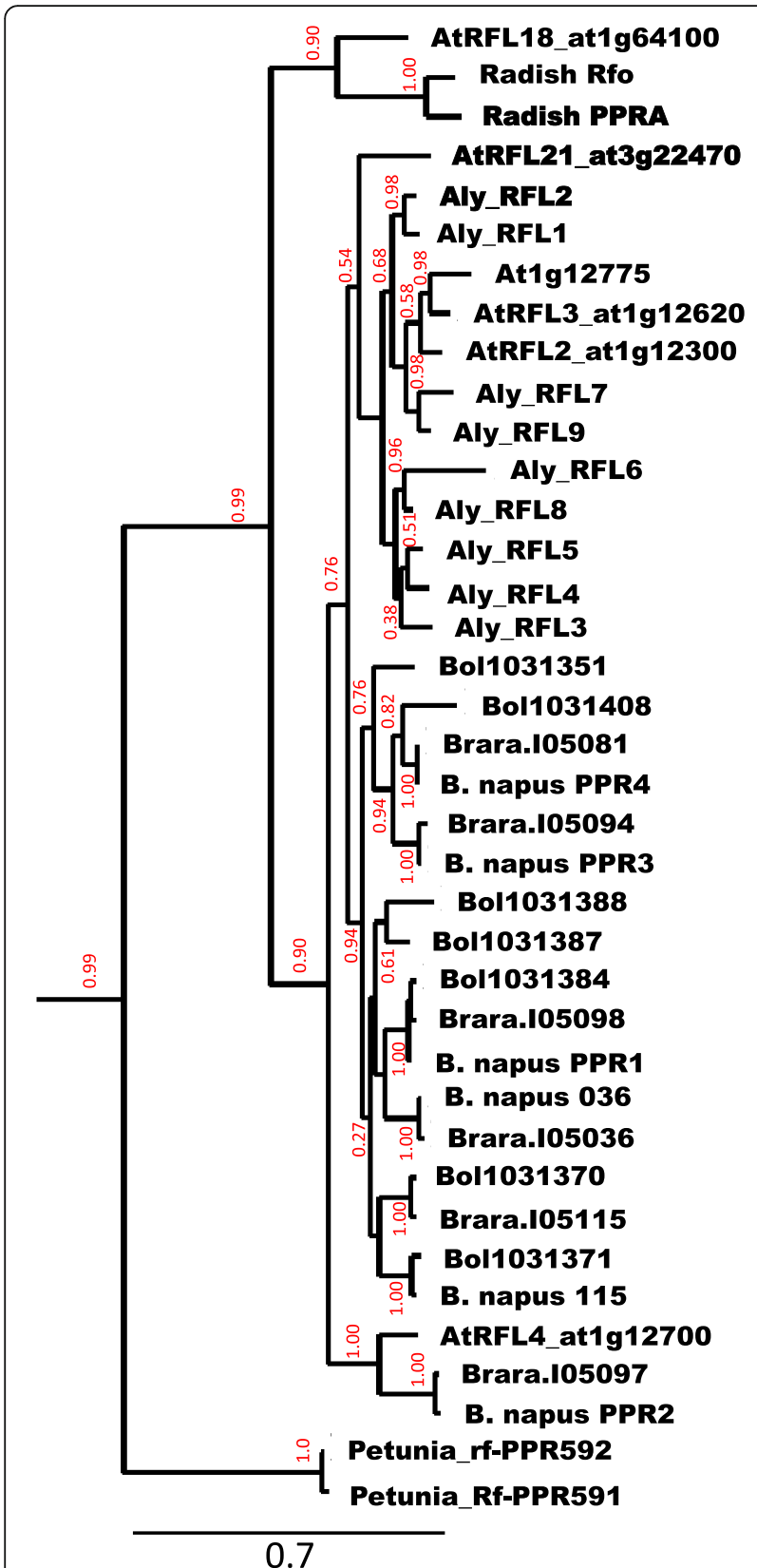

Fig. 7 Phylogeny of Brassica and Arabidopsis PPR proteins. A maximum likelihood tree was generated by the PhyML resource using as input the sequences of known dicot restorer proteins and related orthologs, the predicted protein sequences of $A$. thaliana subgroup 1 RFL proteins, A. lyrata proteins predicted from our analysis of the targeted region enriched in subgroup 1 RFL proteins [22], and the B. napus, B. rapa and B. oleracea RFL proteins of the Brassica Rf-region. Numbers in red flanking each node indicate bootstrap values

PPR4 and its positional B. oleracea counterpart, Bol031408. An interesting situation is observed among the proteins encoded by Brara.I05115 and the genes at the corresponding positions in the B. oleracea and
B. napus genomes, B. oleracea Bol03170 and Bol03171 and $B n$ 115. Although the Rf-region is derived from a $B$. rapa (A genome) ancestor, the $B$. napus and $B$. rapa 115 proteins each group with a different $B$. oleracea protein. Conceivably, orthologs of both genes were present in the common ancestor of the sequenced varieties of B. napus and B. oleracea, and different orthologs were lost during the subsequent evolution of the two A genome forms.

Our manual annotation of the portion of $A$. lyrata genome enriched in subgroup $1 R F L$ genes [22] led to the identification of 10 distinct genes. AlyRFL1-10, one of which (AlyRFL10) had too few PPR domains to merit inclusion in the phylogenetic tree. The two $A$. lyrata genes located at the position of AtRFL2/At1g12300 formed a distinct clade most closely related to the group of $A$. thaliana RFL genes encompassing AtRFL2 as well as the closely related genes AtRFL3/At1g12620 and At1g12775. The position of these two lyrata genes in the tree is consistent with a model in which they arose through a tandem duplication of an AtRFL2-like gene in an ancestral Arabidopsis genome, as proposed above. Similarly, AlyRFL3, AlyRFL4 and AlyRFL5 formed a monophyletic group with AlyRFL6 and AlyRFL8, as would be predicted if there were a gene triplication followed by duplication of the three gene set. The exception to this model concerns AlyRFL7, which was predicted to cluster with AlyRFL6 and AlyRFL8 but instead groups with AlyRFL9. Interestingly, although the coding sequence of this AlyRFL7 is more closely related to AlyRFL9 than to AlyRFL6/8, more similarity is observed in the regions upstream and downstream of the gene to the corresponding sequences in the AlyRFL3-5 region. Conceivably, AlyRFL7 underwent a gene conversion event involving an AlyRFL9 like sequence following duplication of the three gene region.

\section{Discussion}

RFL genes are dispersed in the Brassica Rf-region

A characteristic of genomic loci known to encode nuclear restorer proteins is the occurrence of multiple tandemly repeated related $R F L$ genes [10-13, 15, 44]. Of these, only the rice locus encodes multiple Rf genes that suppress different forms of CMS. In contrast to other $R f$ loci, only two $R F L$ genes within the $B$. napus $R f$ locus, PPR1 and PPR2, are found adjacent to one another. Of these two, PPR2 forms a phylogenetic group with AtRFL4 that is distinct from that formed by the other Arabidopsis and Brassica RFL genes and thus represents a different line of evolutionary descent. Interestingly, the sequences flanking AtRFL4 and Brassica PPR2 are similar to one another, indicating that the genes have both descended from an ancestor located in the same genomic position. 


\section{Retrotransposition has played a major role in the proliferation of Brassica Rf-region RFL genes}

Comparison of the $B$. napus $R f$ locus to orthologous segments of related genomes has provided insight into the mechanisms by which this subgroup of RFL genes has expanded during the evolution of of Brassicacea genomes. With the exception of Brassica PPR2, these genes form a monophyletic cluster in the tree of Fig. 7, indicating descent from a common ancestor. None of the B. napus/rapa RFL genes share common 5' or 3' non-coding regions, suggesting that the expansion of this gene family occurred primarily through mechanisms involving retrotransposition. The observation that none of these genes contains an intron is consistent with this view.

Viewing the sequence relatedness among the different encoded proteins, as assessed through the phylogenetic tree, in the context of the positions of the genes on their respective genomes, provides additional insight into the mechanisms through which these genes proliferated. For example, B. oleracea Bol031408 and B. napus/rapa PPR4, cluster together on the tree, are located in corresponding positions on their genomes and have similar flanking sequences indicating these genes have descended from an ancestral gene present at that location in the last common ancestor of the $\mathrm{A}$ and $\mathrm{C}$ genomes. The coding sequences of B. napus/rapa PPR3 form the closest neighboring phylogenetic cluster to these genes, but are present at a different genomic position, aligning with Bol03187/03188 and At1g12775. However, the sequences flanking PPR3, PPR4, Bol03188 and At1g12775 are all dissimilar, indicating that these genes have proliferated in their respective genomes through retrotransposition. Bol03187 and Bol03188 have similar flanking sequences, indicating a segmental duplication, but their coding sequences cluster with a Bol03184 and PPR1, suggesting that this duplication took place following retrotransposition of a PPR1-like gene.

\section{RFL gene proliferation in A. thaliana and A. lyrata}

Apart from AtRFL4, discussed above, the Arabidopsis $R F L$ genes found in genomic regions orthologous to the Brassica $R f$ locus form a monophyletic group, suggesting these genes have descended from ancestor(s) distinct from those that gave rise to the Brassica $R f$ locus $R F L$ genes. Like majority the Brassica Rf-region RFL genes, the three A. thaliana proteins, AtRFL2, AtRFL3 and At1g12775, form a monophyletic cluster (bootstrap support values of $0.7-1.0)$, and are all located at different genomic positions and lack similarity in their flanking regions, indicating that their proliferation took place through retrotransposition events.

As discussed above, segmental duplication appears to have been the primary mechanism driving the duplication of this gene family in the A. lyrata lineage. Consistent with this model, three of the A. lyrata genes, AlyRFL3-5, predicted to be the products of tandem gene triplication, form a distinct cluster. Of the other three gene group predicted to arise from tandem duplication, AlyRFL6-8, AlyRFL6 and AlyRFL8 form a neighboring cluster in the tree, as would be expected if they arose from the duplication of the AlyRFL3-5 region. AlyRFL7, however, clusters with AlyRFL9, which, with the very short AlyRFL10 (not included in the phylogeny), was predicted to have been generated by a distinct duplication event. Interestingly, the sequences surrounding AlyRFL7 are similar to those flanking AlyRFL6 and AlyRFL8, as would be predicted from the segmental duplication model. It seems likely that following the duplication of the three gene region, retrotransposition and homologous recombination of an AlyRFL9-like gene led to the replacement of the coding sequence of the original gene located AlyRFL7 site with a sequence resembling AlyRFL9.

\section{Prioritization of candidates for the Rfn gene}

A key goal of this undertaking has been to identify candidates for the $R f n$ gene and to prioritize these candidates prior to proceeding with experiments aimed at rescuing the CMS and thereby conclusively identifying the gene. Since most restorer genes have been found to be $R f$-like PPR genes and since the $R f n$ locus delimited in our mapping studies is enriched in RFL genes, we deem it most like that one of these genes functions as $R f n$. Several other types of proteins have been suggested to play a role in fertility restoration, most notably Glycine-rich proteins, or GRPs [18, 44]. We did not, however, detect sequences capable of encoding such proteins in the genetically delimited Brassica $R f$ locus. Likely candidates for $R f n$ are therefore limited to the six $R F L$ genes in this region.

It is logical that a restorer gene be expressed in floral tissues and because we were not able to obtain RT-PCR products for $B n 115$ from floral RNA samples of either CMS or fertility restored plants, it seems unlikely to function as $R f n$. BnO36 is situated $100 \mathrm{~kb}$ from the flanking marker $4.4 \mathrm{BB}$ but $300 \mathrm{~kb}$ from the next closest $R F L$ gene and from the single marker that maps within the sequenced BAC and shows complete linkage to $R f n$. It seems unlikely that the five recombination events we observed between $4.4 \mathrm{BB}$ and $R f n$ in the mapping experiments all occurred within the $100 \mathrm{~kb}$ interval between this marker and Bn036. BnO36 is therefore judged not to be a strong candidate for $R f n$. Of the genes within the selected BAC, we found through the analysis of transcripts of these genes that PPR2 contains a premature termination codon in the line used as the $R f n$ parent of the mapping cross. Thus PPR2 is unlikely to function as $R f n$. 
Thus, only PPR1, PPR3 and PPR4 are reasonable candidates for $R f n$. Of these, $P P R 4$ is expressed in the restorer but not CMS parent an expression pattern previously observed in petunia restoring and nonrestoring lines. While it remains possible that PPR4 does not have a restorer function, it is interesting to note that this gene has also been proposed as a candidate for $R f p$ [42] and its absence of expression in the CMS line would explain why lines that can maintain the nap CMS can also serve as maintainers for pol CMS [29], i.e. they can serve as "universal" maintainers in B. napus. A unique and novel situation would arise if, indeed, different alleles of PPR4 can function as restorers of different forms of $\mathrm{CMS}^{2}$.

\section{Conclusions}

At the outset of this study, our goals were to map the $R f n$ region with sufficient resolution that we could further clarify the relationship of this gene with $R f p$, the restorer for the other native CMS system in B. napus, and to identify a limited number of candidate genes that could then be tested for their capacity to rescue the nap CMS trait through transgenic complementation. It became evident that the Brassica $R f$ locus did not contain a group of tandemly repeated $R F L$ genes, as do other characterized nuclear restorer loci, but that it did correspond to one of the A. thaliana and A. lyrata regions identified by Fujii and colleagues [22] that is enriched in a particular subgroup of $R F L$ genes. We then sought a deeper understanding of the sequence relatedness of between RFL proteins encoded in the Brassica $R f$ locus and the Arabidopsis genomes. In particular we wanted to understand how the sequences of these proteins were related to their genomic position, and how this might relate to the mechanisms by which this group of genes evolved.

Our interpretation of the positional relationships among the genes, as illustrated in Fig. 4 and the phylogenetic relationship among the proteins, as illustrated in Fig. 7, is that both segmental duplication and retrotransposition processes each played a role in the evolution of this region, with segmental duplication being primarily responsible for the expansion of the family in A. lyrata, and retrotransposition playing a more major role in the Brassica genomes. Segmental duplication is most easily understood as arising through unequal genetic crossovers, but the mechanisms driving gene retrotransposition in plants are less well characterized. It has been suggested that the exchange of domains between PPR proteins may provide one means of functional diversification for RFL genes, but we were unable to observe any clear evidence for such an event. We did, however, find evidence for RNA-mediated gene conversion in which the coding sequence an AlyRFL9-like gene replaced the original central gene in the duplication involving the AlyRFL6-8 genes.

Regardless of the mechanism through which this gene family has expanded in the different genomes, it is unclear what function many of these genes may have. AtRFL4 or RPF1, is known to specify specific RNA processing events, as do $R f n$ and $R f p$. Post transcriptional processes and transcript stability control play a dominant role in determining the mature transcriptome of plant mitochondria. In this regard endonuclease cleavage provides a 3' hydroxyl substrate for plant mitochondrial polyA polymerase, which targets substrates for degradation via polynucleotide phosphorylase [45]. The combined consequences of relaxed transcription and numerous ORFs of undefined function in plant mitochondria can result in the expression of "toxic" proteins. CMS provides the clearest example of this phenomenon. In this case, the function of the restorer protein can be viewed as specifying a site or sites for endonuclease cleavage that would then destabilize the 5 ' end of the transcript and prevent its translation [29]. The cryptic transcripts that accumulate in mitochondrial polynucleotide phosphorylase mutants can include other ORFs [46], the expression of which may not cause male sterility but may be otherwise detrimental to the organism. The number and types of these ORFs can change rapidly and the transcript degradation system of the mitochondria must evolve rapidly to accommodate this change. It seems possible that this group of RFL proteins has evolved to specify novel endonuclease cleavage events that trigger the degradation of such transcripts.

\section{Methods}

\section{Identification of an Rfn containing BAC}

Primers based on the sequence of the cosmid 2840A3, generated from $R f p$ doubled haploid $B$. rapa containing the $R f p$-linked SNP 12910 [35], were used for PCR-based screening [47] of a BAC library of $B$. napus doubled haploid line DH12075 constructed in the laboratory of Dr. Isobel Parkin, AAFC, Saskatoon, SK, Canada. A single BAC, designated NO202E11, was selected that generated amplification products with over $99 \%$ similarity to cosmid 2840A3. This BAC contained the $R f n$-associated allele of SNP 12910 and was therefore anchored in the $R f n$ genomic region. The purified BAC was nebulized, subjected to dideoxy sequencing and assembled at the McGill University - Génome Québec Innovation Centre.

\section{Annotation of the Rfn containing BAC sequence}

The assembled sequence contained 8 non-overlapping contigs. These contigs were ordered using BLASTN (http://blast.ncbi.nlm.nih.gov/Blast.cgi) against A. thaliana 
and $B$. rapa genome sequences with the assumption that synteny of these genomes should be mainly conserved. Any missing sequences between the contigs and reorientation of the sequences were investigated by using PCR to amplify the regions between the ends of each contig. Annotation of the completed sequence was investigated by first determining possible open reading frames using Genscan (http://genes.mit.edu/GENSCAN.html) (settings: organism: Arabidopsis, suboptimal exon cutoff: 1.0) and Softberry (http://www.softberry.com/ berry.phtml?topic=case_study_plants\&no_menu=on) (settings: organism: Brassica rapa) online-based tools. Subsequent analysis indicated that Softberry was more accurate at predicting ORFs and became the primary analytical tool for this purpose. Each predicted ORF was then used to find presumptive orthologs in the Brassica rapa and Arabidopsis thaliana genomes using the NCBI tool BLAST (http://blast.ncbi.nlm.nih.gov/Blast.cgi). This analysis allowed us to functionally categorize most of the genes within the BAC. Details of that analysis are presented in Additional file 2: Table S2.

\section{Plant growth and fertility scoring}

Seeds from the two parental lines of Brassica napus used in this present study were acquired from Bo Gertsson, Lantmännen Lantbruk, Svalov, Sweden. The 'Karat-Rfn' nap restorer line is of Swedish origin that presents low levels of both erucic acid and glucosinolates. The population consisted of $318 \mathrm{BC}_{1}$ plants derived from an intervarietal cross between single plants from nap CMS 'Bronowski' (rfurfn; nap) and nap restorer 'Karat-Rfn' (RfnRfn; nap) lines. Seeds were plated onto basic Murashige and Skoog (Sigma-Aldridch M5524-10 L) medium and kept at $4{ }^{\circ} \mathrm{C}$ before germination in a chamber under standard light condition (16-h photoperiod, $22^{\circ} / 16{ }^{\circ} \mathrm{C}$ day/ night temperatures) for a week. Seedlings were transferred in pots and grown to maturity in growth chambers or greenhouses under standard conditions (16-h photoperiod, $22^{\circ} / 16^{\circ} \mathrm{C}$ day/night temperatures).

The fertility was assessed by the careful observation of five flowers per plant at least three times during the flowering period. The overall morphology of the flowers was noted as well as the production of pollen. The flower morphology at the earliest stages of development (2-3 days after flowering) was used as the main criteria to determine the fertility of plants segregating for the restoration of the nap CMS. Later in the development, some nap CMS flowers can produce pollen and make phenotyping ambiguous. Flowers from a male-fertility restored plant look identical to those of a fertile maintainer plant while flowers from a nap CMS plant have shrunken petals, and the style of the pistil is longer and often bent. CMS anthers of young flowers also have shorter filaments and no pollen or a reduced amount of pollen. The morphological contrast of young flowers between CMS and normal flowers was sufficient to allow plants carrying a restorer allele to be distinguished from those with only maintainer alleles, even though some CMS plants shed a small amount of pollen, especially in the later stages of development.

\section{Sampling, DNA extraction and SNP analysis}

Two leaf disks from young plants were sampled into 96 well plates. DNA extraction and SNP genotyping was performed on a Sequenome platform by DNA LandMarks, Inc., St-Jean-sur-Richelieau, Quebec, Canada. 45 SNPs from a list of known B. napus polymorphisms, were selected on the basis of their position in of the on $B$. rapa chromosome A09 were screened for presence of polymorphism in B. napus. SNPs that were polymorphic between the parents of the cross were used to screen the entire $\mathrm{BC} 1$ population.

\section{Marker development}

Analysis of the population was conducted in two phases. In the initial phase 293 plants were genotyped and phenotyped to roughly localize the $R f n$ gene. In the second stage, the $B$. rapa genome [48] was used to design ILP [38] and CAPS [39] markers to more precisely localize the gene. $B$. rapa gene sequences within the mapping interval predefined with the SNP analysis were retrieved from the phytozome database (http://phytozome.jgi.doe.gov/pz/portal.html). Primers were designed to amplify the first intron of every 5 genes using the primer3 web-based tool (bioinfo.ut.ee/primer3-0.4.0/). A 35 cycle PCR was performed following the supplier's instruction (NEB M0273S). Size polymorphisms were detected on 2-3\% agarose gels. For the amplification products that did not show length polymorphisms, we attempted to identify CAPS by performing a $2 \mathrm{~h}$ digestion with enzyme HaeIII (recognition site GGCC) and AluI (recognition site AGCT) and running digestion products on a 2-3\% agarose gel. The amplification products for AFLPs and CAPS were cloned using the TOPO-TA cloning kit (Thermofisher K4575-01, Pleasanton, CA, USA). Information on the primers used, polymorphisms in the parent's sequences can be found in Additional file 1: Table S1.

\section{Synteny analysis}

$B$. napus, B. rapa, B. oleracea and A. thaliana genome fragments corresponding to the mapping interval were extracted from different online databases (https:// genomevolution.org/coge/, http://phytozome.jgi.doe.gov/ pz/portal.html, https://www.arabidopsis.org/index.jsp). The online tool PIP Maker (http://pipmaker.bx.psu.edu/ pipmaker/) was used to assess the conservation of 
sequence linearity between the various genomes analyzed. Brassica rapa gene sequences were extracted (http:// phytozome.jgi.doe.gov/pz/portal.html) in order to investigate homologs in A. thaliana (https://www.arabidopsis.org/ index.jsp) and B. napus (https://genomevolution.org/coge/). Confirmation of co-linearity of relative position was obtained using the "Syntenic Gene" Search tool (http://brassicadb.org/brad/searchSyntenytPCK.php) of the BRAD online resource [43]. This analysis allowed the exploration of the synteny between the 3 genomes. Details of the data obtained are presented in Fig. 4.

\section{Identification of candidate genes and comparative genomics}

Restorers of fertility inducing post-transcriptional processing, as $R f n$ does, have been characterized so far as part of the PPR P-type protein family. In order to do an exhaustive search of the $R f n$-region $B$. napus genomic sequence for possible candidate genes, manual annotation of that genomic fragment was necessary. After extraction of the $600 \mathrm{Mb}$ Rfn containing genomic sequence (http://brassicadb.org/cgi-bin/gbrowse/ B.napus_chromosome/), ORF prediction using Softberry (http://www.softberry.com/berry.phtml?topic=case_study_ plants\&no_menu=on) with $B$. rapa prediction parameters was performed. For each ORF, a BLASTP search on the Brassica rapa (BRAD) and Arabidopsis thaliana (TAIR) genomes was performed in order to detect a probable homolog. That allowed gathering of information of the possible function of the predicted gene could be. With each predicted ORF presenting an annotation of PPR protein, PPR domain prediction was performed using TPRpred (http://toolkit.tuebingen.mpg.de/tprpred) with an e-value inclusion of 1e-6 as well as detection of a mitochondrial targeting site using TargetP (http:// www.cbs.dtu.dk/services/TargetP/) (settings: plant organism group, no cutoff). Information on the syntenic regions from B. rapa, B. oleracea and A. thaliana were gathered from Gbrowse of the different genomic databases available for each genome (https://genomevolution.org/ coge/, http://phytozome.jgi.doe.gov/pz/portal.html, https:// www.arabidopsis.org/index.jsp). To identify corresponding genes in the A. lyrata genome, the region of the scaffold 1 assembly unit enriched in subgroup A_1 RFL [22] genes was manually annotated as described above.

\section{Expression of $R f n$ candidate genes}

Expression of six candidate genes in CMS and restored flower buds were assessed RT-PCR. Total RNA extraction was first performed on $100 \mathrm{mg}$ of floral tissue using Trizol (Thermofisher 15596-026, Pleasanton, CA, USA). Homogenization of frozen tissue was performed by grinding in a sterile mortar and pestle and adding $1 \mathrm{~mL}$ of Trizol. The mixture was mixed and then incubated
$5 \mathrm{~min}$ at room temperature in order to allow rough extraction. $200 \mu \mathrm{L}$ of chloroform was then added and the samples were mixed thoroughly for $15 \mathrm{~s}$ before incubation $2 \mathrm{~min}$ at room temperature and $4{ }^{\circ} \mathrm{C}$ centrifugation for $15 \mathrm{~min}$ at $13000 \mathrm{~g}$. The RNA extract in the upper aqueous phase was then precipitated with $0.5 \mathrm{~mL}$ isopropanol and incubated $10 \mathrm{~min}$ at room temperature before centrifuging at $4{ }^{\circ} \mathrm{C}$ at $12,000 \mathrm{~g}$ for $10 \mathrm{~min}$. The supernatant was then rinsed with $1 \mathrm{~mL} 70 \%$ ethanol and dissolved in $100 \mu \mathrm{L}$ of RNAse free water at 55$60{ }^{\circ} \mathrm{C}$ for $10 \mathrm{~min}$. Purification was then carried out with a standard Qiagen RNeasy kit (Cat No./ID 74904) according to the manufacturer's instruction with DNAse treatment as suggested with (CatNo./ID 79254). After nanodrop quantification, reverse transcription was performed using SuperScript II reverse transcriptase according to the manufacturer instructions using $5 \mu \mathrm{g}$ of total RNA. $2 \mu \mathrm{L}$ of cDNA was used for a 35 cycles PCR using NEB Taq polymerase (catalog \# M0273S, New England Biolabs, Ipswich, MA, USA) according to the supplier's instructions with primers as described in Additional file 1: Table S1. No amplification of candidate Bn115 was observed using samples from both the CMS and restored plants, suggesting this gene is not expressed, at least in the tissue analyzed. Multiple primer sets were tested with the same result. To analyze the presence of introns in the gene sequences the cDNA products were cloned using the TOPO-TA cloning kit (Thermofisher K4575-01) and sequencing was performed by ACGT (http://acgtcorp.com/, Toronto, ON, Canada) with the Applied BioSystems (ABI)/Life Technologies 3730xl capillary electrophoresis DNA sequencer. Sequence analysis of the delivered sequencing graphs were performed using the "geneious" program (http://www.geneious.com/).

\section{Phylogenetic analysis}

From the different online databases available (https:// genomevolution.org/coge/, http://phytozome.jgi.doe.gov/ pz/portal.html, https://www.arabidopsis.org/index.jsp), we selected previously characterized restorer of fertilitylike proteins from petunia and radish, and RFL proteins present in the regions syntenic to the $B$. napus $R f$ locus from A. thaliana, B. rapa and B. oleracea. For A. lyrata, sequences from scaffold 1 (between coordinates 4986000 and 5220000 based on the information provided in [22]) were extracted directly from JGI genome browser (http://genome.jgi.doe.gov/cgi-bin/ browserLoad?db=Araly1\&position=scaffold_1:1-100000) and screened for PPR proteins as described in the identification of $R f n$ candidates. The analysis of the A. lyrata sequence was also used for comparative genomics with $A$. thaliana. Protein sequences were appropriately formatted and phylogeny analysis were performed using 
LIRMM web based tool [49] (www.phylogeny.fr) in advanced mode, allowing a less stringent $G$ Blocks selection and 400 boot strap in order to obtain a stable tree.

\section{Endnote}

${ }^{1}$ The $A$. lyrata genome region enriched in A_1 subgroup RFL genes was originally manually annotated by Fujii et al. [22] but it was not possible for us to determine the details of this annotation from the data provided. We therefore do not know the relationships between the A. lyrata RFL genes analyzed in [22] and those of derived from our annotation. For this reason, we have designated our genes and proteins as AlyRFL1, 2 etc. to distinguish them from those of the earlier publication which were designated as AlRFL1, 2 etc.

${ }^{2}$ Subsequent to the submission of this manuscript, Liu and colleagues published evidence that the gene we refer to as PPR4, when isolated from the Rfp genotype, does indeed encode the Rfp gene (Molecular Plant 9:1082-1084, 2016).

\section{Additional files}

Additional file 1: Table S1. Information on molecular markers. (XLS 14 kb) Additional file 2: Table S2. Annotation of B. napus BAC NO202E11. (XLS $20 \mathrm{~kb}$ )

Additional file 3: Table S3. Annotation of the B. napus $600 \mathrm{~kb}$ Rf-region. (XLS $52 \mathrm{~kb}$ )

Additional file 4: Figure S1. Phylogenetic analysis of proteins encoded in the Rf-region of $B$. napus/rapa and the orthologous segment of $A$. thaliana. Numbers in red type indicate bootstrap values. (PPTX 68 kb)

Additional file 5: Figure S2. Complementary DNA (CDNA) sequences of transcripts of B. napus genes PPR1-4. (PDF $36 \mathrm{~kb}$ )

\section{Abbreviations}

CMS: Cytoplasmic male sterility; Mya: Million years ago or Million years before present; PPR: Pentatricopeptide repeat; Rf: Restorer-of-fertility; RFL: Rf-PPR-like Pentatricopeptide

\section{Acknowledgements}

We thank Dr. Isobel Parkin of Agriculture and Agri-Food Canada (AAFC) for providing access to the BAC library constructed from B. napus DH12075 and to Ryan Nantel-Smith for assistance in selecting and performing initial analysis on BAC NO202E11. This work was funded by grants from National Natural Science and Engineering Council (NSERC) of Canada.

\section{Availability of data and materials}

The supporting data is included as Additional files 1, 2, 3, 4 and 5 .

\section{Authors' contributions}

$L G$ was responsible for the analysis of the BAC sequence, all bioinformatic analysis, the analysis of gene expression, assembly of the tables and she assisted in the preparation of the text and figures. GGB supervised the study and assisted in the preparation of the text and figures. Both authors read and approved the final manuscript.

\section{Competing interests}

Neither of the authors has a competing interest.

\section{Consent for publication}

Not applicable.
Ethics approval and consent to participate

Not applicable.

Received: 10 March 2016 Accepted: 23 September 2016

Published online: 26 October 2016

\section{References}

1. Hanson MR, Bentolila S. Interactions of mitochondrial and nuclear genes that affect male gametophyte development. Plant Cell. 2004;16(Suppl):S154-69.

2. Balk J, Leaver CJ. The PET1-CMS mitochondrial mutation in sunflower is associated with premature programmed cell death and cytochrome $c$ release. Plant Cell. 2001;13(8):1803-18.

3. Sabar M, Gagliardi D, Balk J, Leaver CJ. ORFB is a subunit of F1FO-ATP synthase: insight into the basis of cytoplasmic male sterility in sunflower. EMBO Rep. 2003;4(4):381-6.

4. Luo D, Xu H, Liu Z, Guo J, Li H, Chen L, Fang C, Zhang Q, Bai M, Yao N, et al. A detrimental mitochondrial-nuclear interaction causes cytoplasmic male sterility in rice. Nat Genet. 2013:45(5):573-7.

5. Charlesworth D. A further study of the problem of the maintenance of females in gynodioecious species. Heredity. 1981;46(FEB):27-39.

6. Budar F, Touzet P, De Paepe R. The nucleo-mitochondrial conflict in cytoplasmic male sterilities revisited. Genetica. 2003:117(1):3-16.

7. Delph LF, Touzet P, Bailey MF. Merging theory and mechanism in studies of gynodioecy. Trends Ecol Evol. 2007;22(1):17-24.

8. Li XQ, Jean M, Landry BS, Brown GG. Restorer genes for different forms of Brassica cytoplasmic male sterility map to a single nuclear locus that modifies transcripts of several mitochondrial genes. Proc Natl Acad Sci U S A. 1998;95(17):10032-7.

9. Touzet P, Budar F. Unveiling the molecular arms race between two conflicting genomes in cytoplasmic male sterility? Trends Plant Sci. 2004;9(12):568-70.

10. Bentolila S, Alfonso AA, Hanson MR. A pentatricopeptide repeat-containing gene restores fertility to cytoplasmic male-sterile plants. Proc Natl Acad Sci U S A. 2002;99(16):10887-92.

11. Brown GG, Formanová $N$, Jin $H$, Wargachuk $R$, Dendy C, Patil P, Laforest M, Zhang J, Cheung WY, Landry BS. The radish Rfo restorer gene of Ogura cytoplasmic male sterility encodes a protein with multiple pentatricopeptide repeats. Plant J. 2003;35(2):262-72

12. Koizuka N, Imai R, Fujimoto H, Hayakawa T, Kimura Y, Kohno-Murase J, Sakai T, Kawasaki S, Imamura J. Genetic characterization of a pentatricopeptide repeat protein gene, orf687, that restores fertility in the cytoplasmic male-sterile Kosena radish. The Plant Journal: For Cell and Molecular Biology. 2003;34(4):407-15.

13. Desloire S, Gherbi H, Laloui W, Marhadour S, Clouet V, Cattolico L, Falentin C, Giancola S, Renard M, Budar F, et al. Identification of the fertility restoration locus, Rfo, in radish, as a member of the pentatricopeptide-repeat protein family. EMBO Rep. 2003;4(6):588-94.

14. Kazama T, Toriyama K. A pentatricopeptide repeat-containing gene that promotes the processing of aberrant atp6 RNA of cytoplasmic male-sterile rice. FEBS Lett. 2003:544(1-3):99-102.

15. Akagi H, Nakamura A, Yokozeki-Misono Y, Inagaki A, Takahashi H, Mori K, Fujimura T. Positional cloning of the rice Rf-1 gene, a restorer of BT-type cytoplasmic male sterility that encodes a mitochondria-targeting PPR protein. Theor Appl Genet. 2004;108(8):1449-57.

16. Klein RR, Klein PE, Mullet JE, Minx P, Rooney WL, Schertz KF. Fertility restorer locus Rf1 of sorghum (Sorghum bicolor L.) encodes a pentatricopeptide repeat protein not present in the colinear region of rice chromosome 12. Theor Appl Genet. 2005;111(6):994-1012.

17. Wang ZH, Zou YJ, Li XY, Zhang QY, Chen L, Wu H, Su DH, Chen YL, Guo JX, Luo D, et al. Cytoplasmic male sterility of rice with boro II cytoplasm is caused by a cytotoxic peptide and is restored by two related PPR motif genes via distinct modes of mRNA silencing. Plant Cell. 2006;18(3):676-87.

18. Hu J, Wang K, Huang WC, Liu G, Gao Y, Wang JM, Huang Q, Ji YX, Qin XJ Wan L, et al. The Rice Pentatricopeptide Repeat Protein RF5 Restores Fertility in Hong-Lian Cytoplasmic Male-Sterile Lines via a Complex with the Glycine-Rich Protein GRP162. Plant Cell. 2012;24(1):109-22.

19. Small ID, Peeters N. The PPR motif - a TPR-related motif prevalent in plant organellar proteins. Trends Biochem Sci. 2000;25(2):46-7.

20. Lurin C, Andres C, Aubourg S, Bellaoui M, Bitton F, Bruyere C, Caboche M, Debast C, Gualberto J, Hoffmann B, et al. Genome-wide analysis of 
Arabidopsis pentatricopeptide repeat proteins reveals their essential role in organelle biogenesis. Plant Cell. 2004;16(8):2089-103.

21. Schmitz-Linneweber C, Small I. Pentatricopeptide repeat proteins: a socket set for organelle gene expression. Trends Plant Sci. 2008;13(12):663-70.

22. Fujii S, Bond CS, Small ID. Selection patterns on restorer-like genes reveal a conflict between nuclear and mitochondrial genomes throughout angiosperm evolution. Proc Natl Acad Sci U S A. 2011;108(4):1723-8.

23. Geddy R, Brown GG. Genes encoding pentatricopeptide repeat (PPR) proteins are not conserved in location in plant genomes and may be subject to diversifying selection. BMC Genomics. 2007;8:130.

24. Foxe JP, Wright SI. Signature of Diversifying Selection on Members of the Pentatricopeptide Repeat Protein Family in Arabidopsis lyrata. Genetics. 2009;183(2):663-72.

25. Singh M, Brown GG. Suppression of cytoplasmic male sterility by nuclear genes alters expression of a novel mitochondrial gene region. Plant Cell. 1991;3(12):1349-62.

26. L'Homme Y, Stahl RJ, Li XQ, Hameed A, Brown GG. Brassica nap cytoplasmic male sterility is associated with expression of a mtDNA region containing a chimeric gene similar to the pol CMS-associated orf224 gene. Curr Genet. 1997;31(4):325-35

27. Singh $M$, Hamel N, Menasaa R, Li X-Q, Young B, Jean M, Landry BS, Brown GG. Nuclear Genes Associated With a Single Brassica CMS Restorer Locws Influence Transcripts of Three Different Mitochondrial Gene Regions. Genetics. 1996;143(1):505-16.

28. Menassa R, El-Rouby N, Brown GG. An open reading frame for a protein involved in cytochrome c biogenesis is split into two parts in Brassica mitochondria. Curr Genet. 1997;31(1):70-9.

29. Brown $\mathrm{G}$. Unique aspects of cytoplasmic male sterility and fertility restoration in Brassica napus. J Hered. 1999;90(3):351-6.

30. Town CD, Cheung F, Maiti R, Crabtree J, Haas BJ, Wortman JR, Hine EE, Althoff R, Arbogast TS, Tallon LJ. Comparative genomics of Brassica oleracea and Arabidopsis thaliana reveal gene loss, fragmentation, and dispersal after polyploidy. Plant Cell. 2006;18(6):1348-59.

31. Moghe GD, Hufnagel DE, Tang H, Xiao Y, Dworkin I, Town CD, Conner JK, Shiu S-H. Consequences of whole genome triplication as revewaled by comparative genomic analyses of the wild radish Raphanus raphanistrum and three other Brassicacea species. Plant Cell. 2014;26(5):1925-37.

32. Parkin IA, Gulden SM, Sharpe AG, Lukens L, Trick M, Osborn TC, Lydiate DJ. Segmental structure of the Brassica napus genome based on comparative analysis with Arabidopsis thaliana. Genetics. 2005;171(2):765-81.

33. Cheung F, Trick M, Drou N, Lim YP, Park J-Y, Kwon S-J, Kim J-A, Scott R, Pires JC, Paterson AH. Comparative analysis between homoeologous genome segments of Brassica napus and its progenitor species reveals extensive sequence-level divergence. Plant Cell. 2009;21(7):1912-28.

34. Formanová N, Li X-Q, Ferrie AM, DePauw M, Keller WA, Landry B, Brown GG. Towards positional cloning in Brassica napus: generation and analysis of doubled haploid B. rapa possessing the B. napus pol CMS and Rfp nuclear restorer gene. Plant Mol Biol. 2006;61(1-2):269-81.

35. Formanová N, Stollar R, Geddy R, Mahe L, Laforest M, Landry BS, Brown GG. High-resolution mapping of the Brassica napus Rfp restorer locus using Arabidopsis-derived molecular markers. TAG Theoretical and applied genetics Theoretische und angewandte Genetik. 2010;120(4):843-51.

36. Fan Z, Stefansson B. Influence of temperature on sterility of two cytoplasmic male-sterility systems in rape (Brassica napus L.). Can J Plant Sci. 1986;66(2):221-7.

37. Fan Z, Stefansson B, Sernyk J. Maintainers and restorers for three malesterility-inducing cytoplasms in rape (Brassica napus L.). Can J Plant Sci. 1986;66(2):229-34

38. Wang X, Zhao X, Zhu J, Wu W. Genome-wide investigation of intron length polymorphisms and their potential as molecular markers in rice (Oryza sativa L.). DNA Res. 2006;12(6):417-27.

39. Konieczny A, Ausubel FM. A procedure for mapping Arabidopsis mutations using co-dominant ecotype-specific PCR-based markers. Plant J. 1993;4(2):403-10.

40. Chalhoub B, Denoeud F, Liu S, Parkin IA, Tang H, Wang X, Chiquet J, Belcram H, Tong C, Samans B. Early allopolyploid evolution in the post-Neolithic Brassica napus oilseed genome. Science (New York, NY). 2014;345(6199):950-3.
41. Hölzle A, Jonietz C, Törjek O, Altmann T, Binder S, Forner J. A restorer of fertility-like PPR gene is required for $5^{\prime}$-end processing of the nad4 mRNA in mitochondria of Arabidopsis thaliana. Plant J. 2011;65(5):737-44.

42. Liu Z, Liu P, Long F, Hong D, He Q, Yang G. Fine mapping and candidate gene analysis of the nuclear restorer gene Rfp for pol CMS in rapeseed (Brassica napus L.). Theor Appl Genet. 2012;125(4):773-9.

43. Cheng F, Liu S, Wu J, Fang L, Sun S, Liu B, Li P, Hua W, Wang X. BRAD, the genetics and genomics database for Brassica plants. BMC Plant Biol. 2011;11(1):136.

44. Itabashi E, Iwata N, Fujii S, Kazama T, Toriyama K. The fertility restorer gene, Rf2, for Lead Rice-type cytoplasmic male sterility of rice encodes a mitochondrial glycine-rich protein. Plant J. 2011;65(3):359-67.

45. Holec S, Lange H, Canaday J, Gagliardi D. Coping with cryptic and defective transcripts in plant mitochondria. Biochimica et Biophysica Acta (BBA)-Gene Regulatory Mechanisms. 2008;1779(9):566-73.

46. Holec S, Lange H, Kühn K, Alioua M, Börner T, Gagliardi D. Relaxed transcription in Arabidopsis mitochondria is counterbalanced by RNA stability control mediated by polyadenylation and polynucleotide phosphorylase. Mol Cell Biol. 2006;26(7):2869-76.

47. Farrar K, Donnison IS. Construction and screening of BAC libraries made from Brachypodium genomic DNA. Nat Protoc. 2007;2(7):1661-74.

48. Wang $X$, Wang H, Wang J, Sun R, Wu J, Liu S, Bai Y, Mun J-H, Bancroft I, Cheng $F$, et al. The genome of the mesopolyploid crop species Brassica rapa. Nat Genet. 2011:43(10):1035-9.

49. Dereeper A, Guignon V, Blanc G, Audic S, Buffet S, Chevenet F, Dufayard J-F, Guindon $\mathrm{S}$, Lefort $\mathrm{V}$, Lescot M. Phylogeny. fr: robust phylogenetic analysis for the non-specialist. Nucleic Acids Res. 2008;36 suppl 2:W465-9.

\section{Submit your next manuscript to BioMed Central and we will help you at every step:}

- We accept pre-submission inquiries

- Our selector tool helps you to find the most relevant journal

- We provide round the clock customer support

- Convenient online submission

- Thorough peer review

- Inclusion in PubMed and all major indexing services

- Maximum visibility for your research

Submit your manuscript at www.biomedcentral.com/submit 